\title{
Associações entre Sinais Precoces de Autismo, Atenção Compartilhada e Atrasos no Desenvolvimento Infantil
}

\author{
Livia da Conceição Costa Zaqueu ${ }^{1}$ \\ Maria Cristina Triguero Veloz Teixeira \\ Universidade Presbiteriana Mackenzie \\ Felipe Alckmin-Carvalho \\ Universidade de São Paulo \\ Cristiane Silvestre de Paula \\ Universidade Presbiteriana Mackenzie
}

\begin{abstract}
RESUMO - Os Transtornos do Espectro do Autismo (TEA) costumam comprometer o funcionamento adaptativo e desenvolvimento psicossocial na infância. O objetivo deste estudo foi buscar associações entre: sinais precoces dos TEA, falhas na atenção compartilhada-AC e atrasos de desenvolvimento. Participaram do estudo 92 crianças (16-24 meses) de cinco creches de Barueri-SP. Instrumentos utilizados: Development Screening Test-DENVER-II(desenvolvimento neuropsicomotor), Modified Checklist for Autism in Toddlers-M-CHAT (sinais precoces de TEA), Pictorial Infant Communication Scales-PICS (comunicação social). Identificou-se $28,3 \%$ de atrasos no desenvolvimento neuropsicomotor. Cinco crianças apresentaram sinais precoces dos TEA; todas falharam nas provas de AC (PICS). Nas crianças que apresentaram sinais indicativos de TEA, os déficits mais comuns foram relacionados à atenção compartilhada, área que deve ser privilegiada em avaliações precoces.
\end{abstract}

Palavras-chave: desenvolvimento infantil, transtorno do espectro do autismo, comunicação social, atenção compartilhada

\section{Associations Among Early Signs of Autism, Joint Attention and Developmental Delays in Children}

\begin{abstract}
The Autism Spectrum Disorders (ASD) usually impair adaptive functioning and psychosocial development in childhood. The purpose of this study was to investigate associations among early signs of ASD, failures in joint attention (JA) and developmental delays. Participants were 92 children (16-24 months of age) from five day-care centers in BarueriSP. Assessment instruments used: Development Screening Test-DENVER-II (child development); Modified Checklist for Autism in Toddlers-M-CHAT (screening for ASD); Pictorial Infant Communication Scales-PICS (social communication). Developmental delays were observed in $28.3 \%$ of the children. Five children showed early signs of ASD, all with deficits in joint attention. Since among children with early signs of ASD, the most common deficits were related to joint attention, this field should be privileged in early evaluations.
\end{abstract}

Keywords: child development, autism spectrum disorders, social communication, joint attention

O processo de desenvolvimento infantil inicia-se na vida intrauterina sob a influência de diferentes fatores biológicos e ambientais. Após o nascimento da criança é essencial o monitoramento de diferentes indicadores do desenvolvimento nos aspectos de psicomotricidade, funções sensoriais, linguagem, comunicação, cognição e funcionamento sócio adaptativo. Esse monitoramento é essencial, pois decorrente dele poderão ser conduzidas estimulações precoces para a promoção de um desenvolvimento típico, detecção de fatores de risco para problemas de desenvolvimento, assim como identificação de transtornos do neurodesenvolvimento para iniciar as respectivas intervenções precoces (Centers for Disease Control and Prevention, 2014; Zeppone, Volpon, \& Del Ciampo, 2012). Assim, considera-se que a identificação precoce de alterações em algum desses aspectos constitui um tipo de intervenção preventiva em relação a outros problemas físicos e mentais (Lauritsen, 2013).

1 Endereço para correspondência: Programa de Pós-Graduação em Distúrbios do Desenvolvimento, Universidade Presbiteriana Mackenzie, Rua da Consolação, 896, Consolação, São Paulo, Brasil. CEP: 01.302-907.E-mail: disturbios.pos@mackenzie.br
Uma revisão de literatura de publicações brasileiras entre 2000 e 2011 sobre o tema monitoramento do desenvolvimento infantil indicou que há muito a ser feito nessa área devido a diversos aspectos que vão desde o despreparo dos pediatras até problemas na prática clínica decorrente do uso inadequado de instrumentos de detecção precoce no Brasil (Zeppone et al., 2012). Nessa revisão os autores citam as seguintes evidências como exemplos de necessidades no contexto brasileiro: carência de programas nacionais para vigilância do desenvolvimento neuropsicomotor infantil, falhas na formação e prática clínica de médicos pediatras para detectar atrasos de desenvolvimento, currículos básicos de graduação em Medicina e especialidades de Pediatria e Saúde da Família com escassez de conteúdos dedicados a temas do desenvolvimento infantil e falta de investimento nesses assuntos por parte do Ministério da Saúde (Zeppone et al., 2012).

De forma geral, as avaliações regulares na infância devem ser compreensivas, incluindo medidas de atrasos nos diferentes níveis de funcionamento, como em domínios da comunicação, motricidade, cognição, sociabilidade, 
funcionamento adaptativo, comportamental, sempre considerando os componentes genéticos, assim como história familiar e social da criança (Saulnier, Quiembach, \& Klin, 2011).

Especificamente no caso de crianças com sinais sugestivos de Transtornos do Espectro do Autismo (TEA), equipes de saúde devem estar preparadas para verificar criteriosamente a linha de base de habilidades da criança incluindo os perfis de desenvolvimento, cognição, comunicação, sensorialidade, motricidade e comportamento (Ministério da Saúde, 2012; Saulnier et al., 2011). Na medida em que o profissional esteja melhor preparado para identificar sinais de TEA, a criança poderá ser avaliada precocemente para confirmação ou descarte do diagnóstico e, sequencialmente, proceder com um protocolo de assistência de acordo com o problema que for identificado. No Brasil, nota-se que os profissionais da saúde da atenção primária precisariam ser mais bem capacitados para reconhecer queixas relacionadas aos TEA em idades precoces, ao mesmo tempo em que há uma escassez de serviços públicos especializados na assistência as crianças com TEA (Fatori, Evans-Lacko, Bordin, \& Paula, 2012; Lowenthal, 2013; Paula, Lauridsen-Ribeiro, Wissow, Bordin, \& Evans-Lacko, 2012; Teixeira et al., 2010). Profissionais bem treinados, tanto para o diagnóstico precoce de TEA, quanto para a assistência, impactam positivamente no desenvolvimento da criança. Diferentes tipos de intervenções precoces têm mostrado evidências de ganhos expressivos em indicadores de funcionamento cognitivo, comunicação e interação social, assim como, no funcionamento socioadaptativo dessas crianças (Oono, Honey, \& McConachie, 2013).

Diversos estudos (Ozonoff et al., 2010) têm comprovado que sintomas de TEA, geralmente, são passíveis de identificação a partir dos 12 meses de idade, tornando-se mais estáveis entre os 18 e 24 meses. Por outro lado, a identificação de TEA nos primeiros dois anos de vida continua representando um desafio para as equipes de saúde em todo o mundo, incluindo as brasileiras (Brentani et al., 2013). Alguns marcadores no desenvolvimento têm contribuído substancialmente para a discriminação de crianças com e sem TEA nos primeiros anos de vida, com destaque para falhas nas habilidades de Atenção Compartilhada (AC) (Carvalho, Paula, Teixeira, Zaqueu \& Dantino, 2013; Montenegro, 2007; Montenegro, 2009; Ozonoff et al., 2008).

A AC pode ser definida como o compartilhamento de experiência de um indivíduo com um parceiro social, sendo essa, portanto, uma relação triádica (Montenegro, 2009; Mundy et al., 2007). As habilidades de AC começam a ser observadas por volta dos seis meses de idade em bebês com desenvolvimento típico e se estabelecem plenamente até o final do primeiro ano de vida (Montenegro, 2009). A AC é composta por três comportamentos: i. Resposta à $\mathrm{AC}-\mathrm{RAC}$, ii. Iniciação da AC - IAC e iii. Iniciação de Comportamento de Solicitação - ICS. A RAC se refere à capacidade de seguir a direção do olhar e dos gestos de outras pessoas. A IAC trata da capacidade de usar a direção do olhar e os gestos para direcionar a atenção espontaneamente compartilhando experiências, enquanto que a ICS se refere à habilidade da pessoa em usar o olhar e os gestos para pedir a ajuda de um parceiro social com a intenção de obter um objeto ou evento (Montenegro, 2009; Mundy \& Acra, 2006).

Segundo a quinta edição do Manual de Classificação Estatística dos Transtornos Mentais DSM-5 (APA, 2013), os TEA são transtornos do neurodesenvolvimento que afetam em níveis variados habilidades de interação/e comunicaçãosocial e repertórios de comportamento, estes últimos caracterizados fundamentalmente por interesses restritos e padrões comportamentais repetitivos. Pela abrangência das áreas de desenvolvimento afetadas nos TEA, verifica-se comprometimento, geralmente severo, do funcionamento adaptativo dessas crianças. Por isso, a identificação precoce e implantação de ações de intervenção desde a idade préescolar são bastante recomendadas, já que intervenções precoces, intensivas, de longo prazo e que levem em conta as potencialidades/necessidades de cada criança, têm confirmado melhor prognóstico dos casos (Eldevik et al., 2009; Fernell, Eriksson, \& Gillberg, 2013; Lauritsen, 2013). Entre as intervenções com evidência de eficácia, destacamse as com início precoce do tipo comportamental (Eldevik et al., 2009, Virués-Ortega, 2010) e aquelas direcionadas ao desenvolvimento de habilidades de comunicação e AC (Bosa, 2006), pois elas têm revelado bons resultados principalmente em termos de desenvolvimento cognitivo, de linguagem e em habilidades sociais (Eldevik et al., 2009, Virués-Ortega, 2010).

Nas últimas décadas, a creche se tornou um dos principais locais de agrupamento de crianças brasileiras menores de três anos de idade (Pacheco \& Dupret, 2004), por isso, tem sido considerada um dos locais mais propícios para intervenção na primeira infância. O tempo de permanência dessas crianças em creches permite a observação do seu processo de interação social, favorecendo a identificação de déficits no desenvolvimento infantil incluindo falhas na AC, assim como de outros sinais precoces de TEA. Portanto, propostas de avaliação precoce de atrasos de desenvolvimento no contexto das creches poderão beneficiar à criança, não apenas para o diagnóstico, mas também para a implantação precoce de ações de intervenção. O objetivo deste estudo foi buscar possíveis associações entre sinais precoces de TEA, atrasos de desenvolvimento infantil e falhas nas habilidades de atenção compartilhada.

\section{Método}

Local e participantes: o estudo foi realizado na cidade de Barueri, região metropolitana de São Paulo, localizada a $29 \mathrm{~km}$ da capital. Até o final de 2009, momento da coleta de dados deste estudo, estavam em funcionamento 21 creches públicas municipais em Barueri, totalizando atendimento a 4.502 crianças. Desse universo, foi extraída a amostra de conveniência deste estudo em cinco dessas creches. Numa sexta creche, foi realizado o estudo-piloto. Os critérios de inclusão na amostra foram assiduidade às creches e faixa etária de 16 a 24 meses. Para avaliar a assiduidade foi verificada a frequência das crianças à creche, não podendo ter acima de quatro faltas por semana, mesmo que o motivo da falta fosse justificado pelo responsável. Na aplicação dos instrumentos, foram considerados alguns critérios de 
exclusão transitórios, como período de adaptação à rotina da creche (por meio de consulta ao prontuário/ficha da matrícula; caso estivessem no período de adaptação na creche ou com alguma doença, as crianças eram temporariamente excluídas da amostra). Se durante a aplicação dos instrumentos, a criança demonstrasse sonolência, cansaço, medo/receio, a aplicação era transferida para outra data.

A amostra final do estudo foi composta de 92 crianças (sendo 52,2\% meninos), todos na faixa etária de 16 a 24 meses (média 21,78; + 1,97 meses). A maioria das crianças (89\%) estava na faixa etária de 21 a 24 meses, possuía cor da pele branca $(62,0 \%)$ e havia nascido de parto cesáreo $(52,9 \%)$, conforme dados apresentados na Tabela 1.

Tabela 1 Perfil de crianças frequentadores de cinco creches públicas de Barueri-SP $(N=92)$

\begin{tabular}{lcc}
\hline \multicolumn{1}{c}{ Características Gerais da Criança } & $\mathbf{n ( \% )}$ \\
\hline Faixa Etária & $16-24$ meses & $92(100,0)$ \\
Gênero & Masculino & $48(52,2 \%)$ \\
& Feminino & $44(47,8 \%)$ \\
Cor da pele * & & \\
& Branca & $57(62,0 \%)$ \\
Parda & $31(33,7 \%)$ \\
Tipo de parto ** & Negra & $3(3,3 \%)$ \\
& Não declarado & $1(1,0 \%)$ \\
& Cesárea & $45(52,9 \%)$ \\
& Normal & $38(44,7 \%)$ \\
& Fórceps & $2(2,4 \%)$ \\
\hline
\end{tabular}

\section{Instrumentos}

i) Escala de Comunicação da Primeira Infância-Pictorial Infant Communication Scales (PICS) avalia AC e Orientação Social (OS) (Delgado, Mundy ,\& Block, 2001). A PICS foi desenvolvida para ser aplicada a pais ou cuidadores de crianças, sendo um questionário com 16 itens ilustrados de comportamentos relacionados à $\mathrm{AC}$, assim divididos: seis itens sobre IAC, seis itens sobre ICS e quatro itens que avaliam RAC. Para cada item existem quatro possibilidades de resposta: nunca $=0$; às vezes $=1$; frequentemente $=2$; e não tenho certeza $=9$. A versão brasileira da PICS contém duas questões extras relacionadas ao comportamento de OS, uma para avaliar "resposta ao chamado do nome" e outra para avaliar "imitação ao gesto de bater palmas" (Montenegro, 2007). No presente estudo, utilizamos essa versão com 18 itens que foi aplicada individualmente às cuidadoras responsáveis por cada uma das crianças nas creches. A pontuação média da PICS é obtida mediante a soma dos pontos de cada subgrupo de respostas (IAC/RAC/ ICS excluindo a resposta não tenho certeza); essa somatória é posteriormente dividida pelo número total de respostas (Delgado et al., 2001; Montenegro, 2007), sendo que quanto mais baixa a pontuação na PICS, maior o comprometimento da criança. Evidências de validade da versão brasileira da PICS foram obtidas segundo estudo Caso-Controle com amostra de 17 crianças com diagnóstico de TEA (idade média 36 meses) e 19 crianças com desenvolvimento típico (idade média 63 meses) (Montenegro, 2007). Neste estudo, verificou-se a existência de diferença estatisticamente significante entre as médias da PICS no grupo de crianças com TEA $(M=4,87 ; D P=1,6)$ em comparação com as de crianças com desenvolvimento típico $(M=6,61 ; D P=1,0)$ $(p<0,001)$. Os testes de concordância entre codificadores revelaram bons resultados: acima de $90 \%$ em todas as provas da PICS. Finalmente, os resultados da análise multivariada (Regressão Logística) apontaram que quanto maior a pontuação da PICS, menor a chance de uma criança ser diagnosticada com TEA.

ii) A Escala para Rastreamento Precoce de Autismo - Modified Checklist for Autism in Toddlers (M-CHAT) (Robins, Fein, Barton,\& Green, 2001) foi desenvolvida no formato autoaplicável para identificar sinais precoces de TEA. Trata-se de um questionário padronizado estruturado contendo 23 itens com respostas do tipo SIM/NÃO, que deve ser respondido por pais e/ou cuidadores de crianças de 16 a 30 meses de idade (Kleinman et al., 2008; Losapio \& Pondé, 2008; Robins et al., 2001). As questões do M-CHAT abarcam quatro áreas: (i) Resposta Social (interesse em outras crianças e imitação), (ii) AC (protodeclarativa e olhar monitorado), (iii) Trazer objetos para mostrar para os pais/ cuidadores e (iv) Responder ao chamado dos pais/cuidadores. O M-CHAT foi validado em estudo com 1.294 crianças norte-americanas, demonstrando, por meio de análise da função discriminatória, sensibilidade de $97 \%$, especificidade de $99 \%$, valor preditivo positivo de $80 \%$ e negativo de $99 \%$ (Robins et al., 2001).Embora, este questionário não tenha sido validado para a população brasileira, foram feitas a tradução e adaptação transcultural (Losapio \& Pondé, 2008) e tem sido amplamente utilizado nacionalmente para rastreamento de sinais precoces de TEA. No presente estudo, membros de nossa equipe aplicaram a M-CHAT a cada uma das cuidadoras das creches responsáveis pelas crianças da amostra.

iii) Teste de Triagem de Desenvolvimento de DENVER (Development Screening Test-II - DENVER II) (Frankenberg, Dodds, Archer, Shapiro, \& Bresnick, 1990) avalia indicadores globais de desenvolvimento a partir de quatro áreas: desenvolvimento neuropsicomotor, pessoal-social, habilidades da motricidade fina, linguagem e habilidades da motricidade grossa. A escala é composta por 125 itens que são distribuídos segundo faixas etárias, permitindo a avaliação de crianças de 0 a 6 anos (Frankenberg et al., 1990). Para cada item há uma folha de reposta onde é representada a porcentagem do quanto a criança é capaz de realizá-lo (25, 50,75 e $90 \%$ ) com base na amostra normativa de crianças americanas (Frankenberg et al., 1990; Magalhaes et al., 2014). A versão original do DENVER II possui bons índices de validade e confiabilidade (0,99 interobservador e 0,9 em tese-reteste), sendo esta validade fundamentada em sua própria padronização e não em sua correlação com outros testes (Vieira, Ribeiro,\& Formiga, 2009). Apesar de não ter sido validado no Brasil, o DENVER II é um dos instrumentos de triagem para avaliação de desenvolvimento mais utilizados 
em estudos nacionais, tendo como uma de suas principais vantagens sua rápida aplicação (Halpern, Giugliane, Victora, Barros, \& Horta, 2000; Magalhaes, Fonseca, Martins, \& Dornelas, 2011; Vieira et al., 2009). Na presente pesquisa, cada criança foi avaliada individualmente, segundo as 23 provas do DENVER II correspondentes a sua faixa etária; assim como foram realizadas entrevistas com suas cuidadoras para obtenção de dados relativos a 14 itens do DENVER II.

iv) Os prontuários/fichas da matrícula das crianças foram consultados para coletar dados relativos a caracterização da amostra, incluindo: idade cronológica em meses, gênero, cor da pele, tipo de parto, prematuridade, acompanhamento pré-natal, cirurgias da criança, escolaridade dos pais, renda familiar, desemprego, tipo de moradia, entre outros.

\section{Procedimentos de coleta de dados e considerações éticas}

A realização desta pesquisa foi aprovada pelo Comitê de Ética em Pesquisa Institucional sob o protocolo 0005.0.272.000-09. Os dados foram coletados na creche durante o horário regular de funcionamento para não afetar a rotina de trabalho. O local de coleta foi uma sala privativa com poucos estímulos visuais e auditivos composta por mesa e cadeira para a pesquisadora e mesa pedagógica com cadeira adequada à criança. Todas as crianças foram avaliadas individualmente mediante observação e a partir do relato verbal das cuidadoras das creches como descrito anteriormente. O tempo de duração foi de aproximadamente 25 a 30 minutos. Durante a avaliação as crianças eram acompanhadas por uma cuidadora da creche, porém a mesma permanecia na sala na condição de acompanhante-observador sem interferir na coleta de dados. A equipe que participou da coleta de dados foi composta por quatro alunos de graduação em Psicologia inseridos em projetos de Iniciação Científica e por uma aluna de mestrado.

\section{Procedimentos de análise de dados}

Os dados coletados foram tabulados no programa SPSS, versão 17.0. Inicialmente foram conduzidas análises descritivas para a verificação da frequência de possíveis atrasos no desenvolvimento infantil e sinais precoces de TEA. Posteriormente, análises bivariadas foram realizadas para a verificação de eventuais associações entre os desfechos clínicos de interesse do estudo (desenvolvimento neuropiscomotor e sinais precoces de TEA) e características sociodemográficas da amostra. Para tanto, foram aplicados o teste do Qui-quadrado e Teste Exato de Fisher, conforme o número de casos analisados. Também foi calculada a razão de chances (Oddsratio - OR), considerando-se sempre o intervalo de confiança (IC) de 95\%. Adotou-se nível de probabilidade de $95 \%(p<0,05)$ para a rejeição das hipóteses de nulidade.

\section{Resultados}

Os resultados globais do Teste de DENVER II evidenciaram que $28,3 \%$ das 92 crianças da amostra apresentaram atrasos de desenvolvimento neuropsicomotor, sendo $61,5 \%$ meninos e 38,5\% meninas. Apesar da frequência de atrasos terem sido maior entre os meninos, essa diferença não foi estatisticamente significante entre os gêneros $(p=$ $0,25)$.

No grupo de crianças que apresentou atraso de desenvolvimento, 53,90\% apresentaram falhas em apenas uma área do desenvolvimento; 30,70\% tiveram falhas em duas áreas e 7,70\% falharam em três ou quatro áreas do desenvolvimento. As falhas mais comuns foram observadas nas atividades mais complexas da área de linguagem e na área pessoal-social. Foram verificadas associações entre atrasos de desenvolvimento neuropsicomotor com certas características sociodemográficas e pessoais da amostra: crianças que eram prematuras e aquelas cujas mães não haviam feito acompanhamento pré-natal tinham mais chances de apresentarem atrasos no desenvolvimento neuropsicomotor que as crianças sem esse histórico, conforme demonstra a Tabela 2.

De acordo com os resultados do instrumento de rastreamento M-CHAT, somente quatro das 92 crianças avaliadas foram classificadas como casos suspeitos para TEA. Três dessas quatro crianças apresentaram concomitantemente atrasos no desenvolvimento neuropsicomotor segundo o Teste DENVER II. Os resultados do M-CHAT também foram associados a características sociodemográficas e pessoais da amostra e mostraram associação estatisticamente significante entre sinais precoces de TEA e as variáveis prematuridade e falta de acompanhamento pré-natal ( $p=$ 0,005 e $p=0,03$, respectivamente) (tabela 3 ). Não foram observadas associações com outras variáveis como ter passado por uma cirurgia $(p=0,581)$, internação $(p=0,27)$ ou por acompanhamento médico $(p=0,40)$, de acordo com a Tabela 3.

Identificaram-se também associações entre os resultados do M-CHAT e os dados obtidos da PICS considerando IAC, ICS e RAC. Associações estatisticamente significantes foram verificadas na pontuação total da PICS $(p=0,02), \operatorname{IAC}(p=$ $0,03)$ e ICS $(p=0,02)$, mas não em relação à $\operatorname{RAC}(p=0,21)$. Outras análises foram conduzidas para explorar possíveis associações entre as habilidades de AC (segundo a PICS) e sinais precoces de TEA (segundo M-CHAT). Para tanto, foram feitas análises bivariadas entre cada item da PICS com cada item do M-CHAT, ocasião em que se observou que as cinco crianças classificadas como prováveis casos positivos para TEA segundo o M-CHAT falharam em seis provas da PICS: (i) Aponta para chamar a atenção quando vê um objeto; (ii) Aponta para indicar interesse em um objeto; (iii) Compartilha um evento com o olhar; (iv) Entrega um objeto quando solicitado; (v) Solicita ajuda para obter um objeto; (vi) Olha quando quer obter um objeto. A falha nessas provas da PICS se mostrou, portanto, significativamente associada com sinais precoces de TEA pelo M-CHAT e a intensidade de cada uma dessas associações pode ser conferida na tabela 4 . 
Tabela 2. Associações entre o Teste de Denver e as variáveis da criança, prematuridade, internação, cirurgia, acompanhamento médico, renda familiar e falta de acompanhamento prénatal da mãe $(N=92)$

\begin{tabular}{|c|c|c|c|c|}
\hline \multirow{2}{*}{$\begin{array}{c}\text { Características } \\
\text { sociodemograficas e pessoais }\end{array}$} & \multicolumn{2}{|c|}{$\begin{array}{l}\text { Desenvolvimento } \\
\text { Neuropsicomotor }\end{array}$} & \multirow{2}{*}{$\begin{array}{c}* \text { OR } \\
(\text { IC } 95 \%)\end{array}$} & \multirow{2}{*}{$* * p$} \\
\hline & $\begin{array}{c}\text { Atrasado } \\
\text { n (\%) }\end{array}$ & $\begin{array}{c}\text { Normal } \\
\text { n(\%) }\end{array}$ & & \\
\hline \multicolumn{5}{|l|}{ Criança } \\
\hline \multicolumn{5}{|l|}{ Prematuridade } \\
\hline Sim & $2(100,0)$ & $0 \quad(0)$ & 3,7 & \multirow{2}{*}{0,02} \\
\hline Não & $24(26,7)$ & $66(73,3)$ & $(2,70-5,30)$ & \\
\hline \multicolumn{5}{|l|}{ Internação } \\
\hline Sim & $4(23,5)$ & $13(76,5)$ & 1,35 & \multirow{2}{*}{0,63} \\
\hline Não & $22(29,3)$ & $53(70,7)$ & $(0,40-5,00)$ & \\
\hline \multicolumn{5}{|l|}{ Cirurgia } \\
\hline Sim & $3(60,0)$ & $2(40,0)$ & 0,24 & \multirow{2}{*}{0,10} \\
\hline Não & $23(26,4)$ & $64(73,6)$ & $(0,04-1,53)$ & \\
\hline \multicolumn{5}{|l|}{ Acompanhamento médico } \\
\hline Sim & $15(27,8)$ & $39(72,2)$ & 1,06 & \multirow{2}{*}{0,90} \\
\hline Não & $27(71,0)$ & $11(28,9)$ & $(0,42-2,65)$ & \\
\hline \multicolumn{5}{|l|}{ Renda familiar } \\
\hline Até 2 salários mínimos & $14(32,6)$ & $29(67,4)$ & 1,50 & \multirow{2}{*}{0,40} \\
\hline 3 ou mais salários mínimos & $12(24,5)$ & $66(73,3)$ & $(0,60-4,00)$ & \\
\hline \multicolumn{5}{|l|}{ Mãe } \\
\hline \multicolumn{5}{|l|}{ Falta de pré-natal } \\
\hline Sim & $3(100,0)$ & $0 \quad(0)$ & 0,30 & \multirow{2}{*}{0,05} \\
\hline Não & $23(25,8)$ & $66(73,3)$ & $(0,18-0,16)$ & \\
\hline
\end{tabular}

Nota. ${ }^{*} \mathrm{OR}=$ oddsratio (razão de chances); IC = Intervalo de Confiança; ${ }^{* *} X^{2}$ (teste QuiQuadrado); $(p<0,05)$.

\section{Discussão}

A identificação de atrasos no desenvolvimento neuropsicomotor e de sinais precoces de TEA abre possibilidades para a compreensão de determinadas variáveis envolvidas nessas condições, assim, contribuindo com a adoção de ações e medidas de prevenção e intervenção.

Os resultados obtidos por meio da aplicação do teste de rastreamento DENVER II mostraram que $28,3 \%$ das 92 crianças com idade de 16 a 24 meses aqui avaliadas apresentaram atrasos no desenvolvimento neuropsicomotor. Essa taxa foi similar à de outros estudos brasileiros que, também, testaram crianças em creches. Em estudo (Halpern et al., 2000) realizado em Pelotas, Rio Grande do Sul (RS), em uma amostra de 1.363 crianças, foi identificado que $34 \%$ dos participantes apresentavam atraso no desenvolvimento neuropsicomotor aos 12 meses de idade, segundo o teste de DENVER II. Posteriormente, resultados semelhantes foram encontrados em estudo conduzido em Canoas, RS (Pilz \& Schermann, 2007), no qual foram avaliadas 197 crianças, com idades entre zero aseis anos, com suspeita de atraso no desenvolvimento neuropsicomotor, com o intuito de estabelecer eventuais associações desses atrasos com fatores ambientais e biológicos. Pilz e Schermann (2007) verificaram uma prevalência de atraso no desenvolvimento neuropsicomotor em $27 \%$ do total da amostra de acordo com o mesmo teste de rastreamento. Outro trabalho realizado na região metropolitana de São Paulo (Paula, 2001), em um bairro de baixa renda do município de Embu com uma amostra de crianças de creches na faixa etária entre 9 e 30 meses $(\mathrm{N}=56)$ identificou atrasos no desenvolvimento mental em $33,9 \%$ da amostra e atrasos no desenvolvimento motor em $29,6 \%$ do grupo.

No presente grupo de participantes a área de desenvolvimento que mostrou maiores prejuízos foi a da linguagem (54,48\%), seguida da área pessoal-social $(17,97 \%)$, da motora fina adaptativa $(14,74 \%)$ e da motora grossa $(12,81 \%)$. Esses números são semelhantes aos encontrados por Biscegli, Polis, Santos \& Vicentin (2007), que apontam a área da linguagem como a mais deficitária em crianças de creches brasileiras. Mesmo em outros ambientes, como em Unidades Básicas de Saúde, a área de linguagem tem sido apontada como a mais afetada entre os atrasos do 
Tabela 3. Associações entre ausência ou presença de sinais precoces de TEA e as variáveis da criança, prematuridade, internação, cirurgia, acompanhamento médico, renda familiar e falta de acompanhamento pré-natal da mãe $(N=92)$

\begin{tabular}{|c|c|c|c|c|}
\hline \multirow{2}{*}{$\begin{array}{c}\text { Características } \\
\text { sociodemograficas e pessoais }\end{array}$} & \multicolumn{2}{|c|}{ Sinais de TEA } & \multirow{2}{*}{$\begin{array}{c}* \text { OR } \\
(\text { IC } 95 \%)\end{array}$} & \multirow{2}{*}{$* * p$} \\
\hline & Ausentes & Presente & & \\
\hline \multicolumn{5}{|l|}{ Criança } \\
\hline \multicolumn{5}{|l|}{ Prematuridade } \\
\hline Sim & $1(50,0)$ & $1(50,0)$ & 0,04 & \multirow{2}{*}{0,05} \\
\hline Não & $86(96,0)$ & $4 \quad(4,0)$ & $(0,002-0,89)$ & \\
\hline \multicolumn{5}{|l|}{ Internação } \\
\hline Sim & $17(100,0)$ & $0(0,0)$ & 0,80 & \multirow{2}{*}{0,27} \\
\hline Não & $70(93,0)$ & $5(7,0)$ & $(0,73-0,89)$ & \\
\hline \multicolumn{5}{|l|}{ Cirurgia } \\
\hline Sim & $5(100,0)$ & $0(0,0)$ & 0,94 & \multirow{2}{*}{0,058} \\
\hline Não & $82(94,0)$ & $5(6,0)$ & $(0,90-0,99)$ & \\
\hline \multicolumn{5}{|l|}{ Acompanhamento médico } \\
\hline Sim & $52(96,0)$ & $2(4,0)$ & & \multirow{2}{*}{0,90} \\
\hline Não & $35(92,0)$ & $3(8,0)$ & & \\
\hline \multicolumn{5}{|l|}{ Renda familiar } \\
\hline Até 2 salários mínimos & $44(90,0)$ & $5(10,0)$ & 1,0 & \multirow{2}{*}{0,06} \\
\hline 3 ou mais salários mínimos & $43(100,0)$ & $0 \quad(0,0)$ & $(0,82-1,00)$ & \\
\hline \multicolumn{5}{|l|}{ Mãe } \\
\hline \multicolumn{5}{|l|}{ Pré-natal } \\
\hline Sim & $85(96,0)$ & $4(4,0)$ & \multirow{2}{*}{$\begin{array}{c}10,62(0,79- \\
143,26)\end{array}$} & \multirow{2}{*}{0,03} \\
\hline Não & $2(67,0)$ & $1(33,0)$ & & \\
\hline
\end{tabular}

Nota $.{ }^{*} \mathrm{OR}=$ oddsratio (razão de chances); IC $=$ Intervalo de Confiança; $* * X^{2}$ (teste Qui-

Quadrado); $(p<0,05)$.

Tabela 4. Associação entre ausência ou presença de sinais precoces de transtorno do Espectro Autista e os resultados da PICS em relação às habilidades de Atenção Compartilhada

\begin{tabular}{|c|c|c|c|c|}
\hline \multirow[b]{2}{*}{ Provas da Pics } & \multicolumn{2}{|c|}{ Sinais de TEA } & \multirow[b]{2}{*}{$\begin{array}{c}\text { *OR } \\
\text { (IC 95\%) }\end{array}$} & \multirow[b]{2}{*}{$* * p$} \\
\hline & $\begin{array}{c}\text { Ausentes } \\
\text { n (\%) }\end{array}$ & $\begin{array}{c}\text { Presentes } \\
\text { n (\%) }\end{array}$ & & \\
\hline $\begin{array}{l}\text { Aponta para chamar a atenção quando vê um objeto } \\
\text { Passou } \\
\text { Falhou }\end{array}$ & $\begin{array}{l}58(66,7) \\
29(33,6)\end{array}$ & $\begin{array}{c}0(0) \\
4(100,0)\end{array}$ & $2,00(1,02-2,34)$ & 0,03 \\
\hline $\begin{array}{l}\text { Aponta para indicar interesse em um objeto } \\
\text { Passou } \\
\text { Falhou }\end{array}$ & $\begin{array}{l}40(60,6) \\
36(39,4)\end{array}$ & $\begin{array}{c}0(0) \\
4(100,0)\end{array}$ & $1,16(1,01-1,31)$ & 0,05 \\
\hline $\begin{array}{l}\text { Compartilha um evento com o olhar } \\
\text { Passou } \\
\text { Falhou }\end{array}$ & $\begin{array}{l}49(56,3) \\
38(47,3)\end{array}$ & $\begin{array}{c}0(0) 4 \\
(100,0)\end{array}$ & $1,13(1,01-1,26)$ & 0,01 \\
\hline $\begin{array}{l}\text { Entrega um objeto quando solicitado } \\
\text { Passou } \\
\text { Falhou }\end{array}$ & $\begin{array}{l}42(48,3) \\
45(51,7)\end{array}$ & $\begin{array}{c}0(0) \\
4(100,0)\end{array}$ & $1,11(1,00-1,20)$ & 0,03 \\
\hline $\begin{array}{l}\text { Solicita ajuda para obter um objeto } \\
\text { Passou } \\
\text { Falhou }\end{array}$ & $\begin{array}{l}48(55,2) \\
39(44,8)\end{array}$ & $\begin{array}{c}0(0) \\
4(100,0)\end{array}$ & $1,13(1,00-1,25)$ & 0,01 \\
\hline $\begin{array}{l}\text { Olha quando quer obter um objeto } \\
\text { Passou } \\
\text { Falhou }\end{array}$ & $\begin{array}{l}55(63,2) \\
32(36,8)\end{array}$ & $\begin{array}{c}0(0) \\
4(100,0)\end{array}$ & $1,60(1,00-1,30)$ & 0,05 \\
\hline
\end{tabular}

Nota. ${ }^{*} \mathrm{OR}=$ oddsratio (razão de chances); IC = Intervalo de Confiança; ** $X^{2}$ (teste Qui-Quadrado); $(p<0,05)$. 
desenvolvimento neuropsicomotor identificados por testes de rastreamento (DumontMethieu \& Fein, 2005; Fernell, 2013; Nobre, Carvalho, Martinez, \& Linhares 2009; Saccani et al., 2007).

Foram investigados fatores sociodemográficos, como renda familiar, escolaridade dos pais, e fatores pessoais dos participantes de amostra, como prematuridade, acompanhamento pré-natal, realização de cirurgia(s), internação hospitalar e acompanhamento médico. Verificaramse associações estatisticamente significantes entre atrasos no desenvolvimento neuropsicomotor e prematuridade $(p=$ $0,02)$ e falta de acompanhamento pré-natal $(p=0,005)$. O estudo brasileiro de Nunes, Sisdelli e Fernandes (1994) também encontrou associação estatisticamente significante entre falta de acompanhamento pré-natal e atrasos de desenvolvimento neuropsicomotor. Paralelamente, o risco aumentado de problemas de desenvolvimento em prematuros também está bem estabelecido na literatura, já que estudo de revisão conduzido por Linhares et al. (2004) descreve diversas pesquisas com delineamento longitudinal confirmando esta associação. Uma das pesquisas citadas nesta revisão se refere ao estudo de Beckwith e Rodning (citado por Linhares et al., 2004) que demonstra que a criança prematura é mais vulnerável a riscos biológicos e psicossociais que as crianças a termo, e que por isso precisam ser regularmente monitoradas para evitar atrasos em seu desenvolvimento. Outros estudos desta mesma revisão trataram dos aspectos psicológicos envolvidos no nascimento de um prematuro. De acordo com Goldberg e Vitto (citado por Linhares et al., 2004), podem haver prejuízos no estabelecimento da relação mãe-bebê pelo afastamento precoce nos casos daqueles que ficam internados em uma Unidade de Terapia Intensiva, enquanto que estudo realizado por Pedromônico (citado por Linhares et al., 2004) indica que em alguns casos, a mãe precisa resignificar a imagem feita durante a gravidez de um bebê absolutamente saudável, o que pode dificultar, em algum nível, o vínculo mãe-bebê.

Sabendo-se que a relação mãe-bebê é relevante para o desenvolvimento emocional da criança, os estudos citados acima indicam que a prematuridade associada a dificuldades no estabelecimento da relação mãe-filho, são fatores que aumentam a chance do aparecimento de problemas no decorrer do desenvolvimento. Portanto, de forma geral, todos estes estudos recomendam que políticas públicas de saúde estimulem o acompanhamento pré-natal visando o decréscimo de partos prematuros, pois estas contribuirão indiretamente para minimizar atrasos no desenvolvimento infantil.

No presente estudo foi verificado que $5,4 \%$ das crianças apresentavam sinais sugestivos de TEA de acordo com o $\mathrm{M}$-CHAT. Essa taxa é superior à relatada em estudos recentes sobre prevalência de TEA nos quais esse percentual varia de $0,6 \%$ a $1 \%$, quando são utilizados instrumentos padrão-ouro que permitem o estabelecimento do diagnóstico de TEA (Center for Disease and Control, 2014; Charman et al., 2005; Elsabbagh et al., 2012; Fombonne, 2006; Fombonne, 2009). Entretanto, o percentual de 5,4\% observado neste estudo é inferior com o encontrado em outras pesquisas que também utilizaram o M-CHAT. É o caso do estudo de Robins (2008), que encontrou uma taxa de $9 \%$ de suspeita de TEA em uma amostra com 4.797 crianças avaliadas durante consultas pediátricas. No entanto, das 466 com suspeita de TEA segundo o M-CHAT, apenas quatro tiveram o diagnóstico de TEA confirmado de acordo com ADOS, a ADI-R e a CARS.

Essas discrepâncias entre dados suspeitos de TEA com uso de instrumentos de rastreamento como o M-CHAT e dados confirmados de TEA de acordo com instrumentos diagnóstico mostra que o M-CHAT tem uma alta sensibilidade, mas baixa especificidade para o transtorno. Daí a necessidade de uma equipe de saúde capacitada para verificar criteriosamente e, mediante instrumentos de avaliação diagnóstica, perfis de desenvolvimento, cognição, fala, linguagem, comunicação, sensorialidade, motricidade e comportamento da criança com suspeita de TEA (Brentani et al., 2013; Ministério da Saúde, 2012). De fato, estudo anterior já havia apontado alta sensibilidade (92\%) do M-CHAT em um grupo de 84 crianças com idades entre 24 e 48 meses (Eaves, Ho, \& Helena, 2006). O M-CHAT é capaz de detectar precocemente número importante de prováveis casos sugestivos de TEA antes da realização de uma avaliação clínica geral exaustiva. Tratando-se de um estudo de rastreamento, os casos identificados como suspeitos neste trabalho precisam de outras avaliações diagnósticas futuras para a confirmação do quadro de TEA. Por outro lado, mesmo que não confirmado o diagnóstico de TEA, essas cinco crianças apresentam atrasos no desenvolvimento em algum nível, sugerindo o acompanhamento e a vigilância do seu desenvolvimento.

Também foram verificadas no presente estudo, associações entre sinais precoces de TEA (M-CHAT) com prematuridade e falta de acompanhamento pré-natal. Esses achados corroboram estudos internacionais que têm apontado risco de problemas pré e perinatais entre crianças com TEA (Kolevzon, Gross, \& Reichenberg, 2007; Limperopoulos et al., 2008). Estudo de revisão feito por Kolevzonet al. (2007) mostra que um dos principais fatores pré-natais e perinatais é a prematuridade e

a falta de acompanhamento pré-natal, além de idade avançada dos pais. Do mesmo modo, outro estudo realizado no Canadá, com uma amostra de 91 crianças nascidas com extremo baixo peso $(<1.500$ gramas $)$, indicou forte associação entre prematuridade e casos suspeitos de TEA (utilizando o instrumento de rastreamento M-CHAT) (Limperopoulos et al., 2008).

No presente estudo verificou-se que os itens do M-CHAT com maior número de falhas foram das áreas de relacionamento social e habilidades de AC (especificamente nos comportamentos de IAC e de ICS representados pelos itens a) aponta para chamar a atenção quando vê um objeto, b) aponta para indicar interesse em um objeto, c) compartilha um evento com o olhar, d) entrega um objeto quando solicitado, e) solicita ajuda para obter um objeto, f) olha quando quer obter um objeto). Dados de outros trabalhos também têm mostrado que aspectos da IAC e da ICS investigados no M-CHAT são bons marcadores de TEA, particularmente os que avaliam se a criança usa o dedo indicador dela para apontar, se traz objetos para mostrar ao cuidador e se acompanha com o olhar quando se aponta um brinquedo (Wong et al., 2004). É importante ressaltar também que prejuízos na área de IAC têm sido consistentemente observados em crianças com TEA desde a fase pré-escolar até 
a adolescência, constituindo-se como um dos principais sinais de TEA (Carvalho et al., 2013; Charman, 2003; Dawson et al., 2004; Montenegro, 2007; Mundy et al., 2007).

O presente estudo apresenta algumas limitações que merecem ser citadas. A amostra foi exclusiva de creches públicas, o que limita a generalização dos resultados para outros contextos. Parte da coleta de dados foi realizada a partir de entrevistas com as cuidadoras das creches, que podem desconhecer detalhes de aspectos comportamentais das crianças participantes do estudo e que por se tratar de uma informação indireta (ao invés da testagem direta das crianças) pode conter imprecisões.

Os instrumentos utilizados no presente estudo são de rastreamento, portanto é preciso deixar claro que não se propõem a estabelecer qualquer tipo de diagnóstico clínico, principalmente em relação aos TEA.

Apesar dessas limitações, essa pesquisa traz interessantes contribuições relativas às primeiras fases de desenvolvimento de crianças, indicando, por exemplo, que atrasos no desenvolvimento podem e devem ser detectados precocemente; que a área da linguagem é uma das mais afetadas; e que existem grupos mais vulneráveis que devem ser priorizados em ações de políticas públicas nos campos da saúde e da educação. Como mencionado anteriormente, avaliações regulares na infância tem sido recomendadas (Saulnier et al., 2011), sendo a creche pública um local interessante para identificação de problemas e subsequente intervenção por ser um dos principais locais de agrupamento de crianças brasileiras menores de três anos de idade (Pacheco \& Dupret, 2004).

Finalmente, é importante ressaltar que na hora da escolha do tipo de intervenção a ser implanta no sistema público, recomenda-se aquelas com evidência de eficácia, já que a literatura tem apontado certos modelos que levam ao melhor prognóstico dos casos (Eldevik et al., 2009; Fernell et al., 2013; Lauritsen, 2013).

\section{Considerações Finais}

O estudo identificou uma taxa de atrasos do desenvolvimento relativamente elevada na amostra, sendo a área da linguagem a mais afetada. A estimulação de habilidades de linguagem nas crianças participantes é recomendável para evitar prejuízos futuros, principalmente antes de seu ingresso na educação formal.

As associações estatisticamente significantes verificadas entre o atraso de desenvolvimento neuropsicomotor e sinais precoces de TEA, com as variáveis prematuridade e falta de acompanhamento pré-natal, alertam para a existência de grupos mais vulneráveis, assim como para a necessidade de vigilância e monitoramento do desenvolvimento dessas crianças.

Os resultados mostraram que entre as crianças com sinais sugestivos de TEA os maiores déficits foram observados nos indicadores de AC, especificamente relacionados ao gesto de apontar para chamar a atenção, mostrar um objeto/evento e solicitar ajuda para pegar um objeto. Esses sinais seriam facilmente identificáveis se os profissionais das creches estivessem capacitados no tema. Dessa maneira, a equipe educacional da creche poderia agir como mediador com as equipes de saúde no levantamento de possíveis suspeitas de crianças com atraso no desenvolvimento ou com suspeita de TEA. Isto permitiria a implantação de intervenções precoces, especificamente relacionadas às habilidades de AC. Além disso, a creche passaria a exercer suas funções de educar e cuidar promovendo uma melhoria das condições da saúde mental infantil, identificando, acompanhando e possibilitando intervenções eficazes para essa população.

\section{Referências}

American Psychiatric Association - APA (2013).Diagnostic and statistical manual of mental disorders (5a ed.).Washington, DC: APA.

Biscegli, T. S., Polis, L. B., Santos, L. M., \& Vicentin, M. (2007). Avaliação do estado nutricional e do desenvolvimento neuropsicomotor em crianças frequentadoras de creche. Revista Paulista de Pediatria, 25(4), 337-342.

Bosa, C. A. (2006). Autismo: intervenções psicoeducacionais. Revista Brasileira Psiquiatria, 28 (Supl I), S47-S53.

Brentani, H., Paula, C. S. D., Bordini, D., Rolim, D., Sato, F., Portolese, J., Pacífico, M.C., \& McCracken, J. T. (2013). Autism spectrum disorders: an overview on diagnosis and treatment. Revista Brasileira de Psiquiatria, 35(Supl I),S62-S72.

Carvalho, F. A., Paula, C. S., Teixeira, M. C. T. V., Zaqueu, L. C. C., \& D'Antino, M. E. F. (2013). Rastreamento de sinais precoces de Transtornos do Espectro do Autismo em crianças de creches de um município de São Paulo. Psicologia: Teoria e Prática, 15(2), 144-154.

Center for Disease Control. (2014). Prevalence of Autism Spectrum Disorder Among Children Aged 8 Years - Autism and Developmental Disabilities Monitoring Network, 11 Sites, United States, 2010. Morbidity and Mortality Weekly Report Surveillance Summary, 63(2), 1-22.

Charman, T. (2003). Why is joint attention a pivotal skill in autism? Philosophical Transactions of the Royal Society of London. Series B: Biological Sciences, 358(1430), 315-324.

Charman, T., Taylor, E., Drew, A., Cockerill, H., Brown, J. A., \& Baird, G. (2005). Outcome at 7 years of children diagnosed with autism at age 2: predictive validity of assessments conducted at 2 and 3 years of age and patterns of symptom change over time. Journal Child Psychology Psychiatry, 46(5), 500-513.

Dawson, G., Toth, K., Abbot, R., Osterling, J., Munson, J., Estes, A., \& Liaw, J. (2004). Early social attention impairments in autism: social orienting, joint attention to distress. Developmental Psychology, 40(2), 271-283.

Delgado, C., Mundy, P., \& Block, J. (2001). Pictorial Infant Communication Scales (PICS), Version 1.3. Miami: University of Miami.

DumontMathieu, T., \& Fein, D. (2001). Screening for autism in young children: the Modified Checklist for Autism in Toddlers (M-CHAT) and other measures. Mental Retardation and Developmental Disabilities Research Reviews, 11(3), 253-262.

Eaves, L., Wingert, H. \& Helena H. (2006). Screening for autism: agreement with diagnosis. Autism, 10(3), 229-242. 
Eldevik, S., Hastings, R. P., Hughes, J. C., Jahr, E., Eikeseth, S., \& Cross, S. (2009). Meta-Analysis of Early Intensive Behavioral Intervention for Children with Autism. Journal of Clinical Child \& Adolescent Psychology, 38(3),439-450.

Elsabbagh, M., Divan, G., Koh, Y. J, Kim, Y. S., Kauchali, S., Marcín, C. \& Fombonne, E. (2012). Global Prevalence of Autism and Other Pervasive Developmental Disorders. Autism Research, 5(3), 160-179.

Fatori, D., Evans-Lacko, S., Bordin, I. A., \& Paula, C. S. (2012). Child mental health care in Brazil: barriers and achievements. Lancet, 379(9812), 16-17.

Fernell, E., Eriksson, M. A., \& Gillberg, C. (2013). Early diagnosis of autism and impact on prognosis: a narrative review. Clinical Epidemiology, 5(1), 33-43.

Fombonne, E. (2009). Epidemiology of pervasive developmental disorders. Pediatric Research, 65(6), 591-598.

Fombonne, E. (2006). Past and future perspectives on autism epidemiology. In S. Moldin \& J. Rubenstein (Eds.), Understanding Autism: From Basic Neuroscience to Treatment. (pp. 25-46). CRC Press, Boca Raton, Florida.

Frankenberg, W. K., Dodds, J., Archer, P., Shapiro, H., \& Bresnick, B. (1990). Denver II Screening Manual. Denver: Denver Development Materials Inc.

Halpern, R., Giugliani, E. R. J., Victora, C. G., Barros, F. C., \& Horta, B. L. (2000). Fatores de risco para suspeita de atraso no desenvolvimento neuropsicomotor aos 12 meses de vida. Jornal de Pediatria, 76(6), 421-428.

Kleinman, J. M., Robins, D. L., Ventola, P. E., Pandey, J., Boorstein, H. C., Esser, E. L., \& Fein, D. (2008). The modified checklist for autism in toddlers: a follow-up study investigating the early detection of autism spectrum disorders. Journal of Autism and Developmental Disorders, 38(5), 827-839.

Kolevzon, A., Gross, R., \& Reicheberg, A. (2007). Prenatal and perinatal risk factors for autism: a review and integration of findings. Archives of Pediatrics and Adolescent Medicine, 161(4), 326-333.

Lauritsen, M. B. (2013). Autism spectrum disorders. European Child \& Adolescent Psychiatry, 22(1), 37-42.

Limperopoulos, C., Bassan, H., Sullivan, N. R., Soul, J. S., Robertson, R. L., Moore, M., Ringer, S. R.,Volpe, J. J., \& Plessis, A. (2008). Positive screening for autism in ex-preterm infants: prevalence and risk factors. Pediatrics, 121(4),758765.

Linhares, M. B. M., Carvalho, A. E. V., Padovani, F. H. P., Bordin, M. B., Martins, I. M. B., \& Martinez, F. E. (2004). Compreensão do fator de risco da prematuridade sob a ótica desenvolvimental. In E. M. Marturano, M. B. M. Linhares, S. D. R. \& Loureiro (Eds.), Vulnerabilidade e proteção: Indicadores na trajetória de desenvolvimento do escolar. (pp. 11-38). São Paulo: Casa do Psicólogo.

Losapio, M. F., \& Pondé, M. P. (2008). Tradução para o português da escala M-CHAT para rastreamento precoce de autismo. Revista de Psiquiatria do Rio Grande do Sul, 30(3), 101-108.

Lowenthal, R (2013). Saúde mental na infância: proposta de capacitação para Atenção Primária. São Paulo: Editora Mackenzie, 102p.

Magalhaes, L. C., Fonseca, K. L., Martins, L. D. T. B., \& Dornelas, L. F. (2011). Desempenho de crianças pré-termo com muito baixo peso e extremo baixo peso segundo o teste Denver-II. Revista Brasileira Saúde Materno Infantil, 11(4), 445-453.
Ministério da Saúde. (2012). Diretrizes de atenção à reabilitação da pessoa com transtornos do espectro do autismo TEA. Retrievedfromhttp://portal.saude.gov.br/404.html. Acesso em 29 de abril de 2014.

Montenegro M. (2009). Atenção Compartilhada. In M. T. Mercadante\& M. C. Rosário (Eds.), Autismo e Cérebro Social (v.1, pp. 75-86). São Paulo: Segmento Farma Editores.

Montenegro, M. (2007). Avaliação e estudo dos comportamentos de orientação social e a atenção compartilhada nos transtornos invasivos do desenvolvimento. (Unpublishedmaster'sthesis). Universidade Presbiteriana Mackenzie, São Paulo.

Mundy, P., Block, J., Delgado, C., Pomares, Y., Van Hecke, A. V., \& Parlade, M. V. (2007). Individual differences and the development of joint attention in infancy.Child Development, 78(3), 938-954.

Mundy, P., \& Acra, F. (2006). Joint attention, social engagement, and the development of social competence. In P. Marshall \& N. Fox (Eds.), The development of social engagement: neurobiological perspectives (pp. 81-117). New York: Oxford University Press.

Nobre, F. D. A., Carvalho, A. E. V., Martinez, F. E., \& Linhares, M. B. M. (2009). Estudo longitudinal do desenvolvimento de crianças nascidas pré-termo no primeiro ano pós-natal. Psicologia: Reflexão e Crítica, 22(3), 362-369.

Nunes, L. R. D. O. P, Sisdelli, R. O., \& Fernandes, R. L. C. (1994). O valor dos testes de bebês e suas implicações para a psicologia do desenvolvimento e educação especial. Cadernos de Educação Especial, 1(2), 107-125.

Oono, I. P., Honey, E. J., \& McConachie, H. (2013). Parent-mediated early intervention for young children with autism spectrum disorders. Cochrane Database, 30(4), 1-98.

Ozonoff, S., Young, G. S., Goldring, S., GreissHess, L., Herrera, A. M., Steele, J., Macari, S., Hapburn, S., \& Rogers, S. J. (2008). Gross motor development, movement abnormalities, and early identification of autism. Journal of Autism and Developmental Disorders, 38(4), 644-656.

Ozonoff, S., Iosif, A. M., Baguio, F., Cook, I. C., Hill, M. M., Hutman, T., \& Young, G. S. (2010). A prospective study of the emergence of early behavioral signs of autism. Journal of the American Academy of Child and Adolescent Psychiatry, 49(3), 256-266.

Pacheco, A. L. P. D. B., \& Dupret, L. (2004). Creche: desenvolvimento ou sobrevivência? Psicologia USP, 15(3), 103-116.

Paula, C. (2001). Atrasos de desenvolvimento mental e motor em crianças de creches de comunidade urbana de baixa renda e fatores de risco associados (Unpublishedmaster'sthesis). São Paulo: Departamento de Psiquiatria da Universal Federal de São Paulo, 147 p.

Paula C. S., Lauridsen-Ribeiro E., Wissow, L., Bordin, I. A., \& Evans-Lacko, S. (2012). How to improve the mental health care of children and adolescents in Brazil: actions needed in the public sector. Revista Brasileira de Psiquiatria, 34(3), 334-351.

Pilz, E. M. L., \& Schermann, L. B. (2007). Determinantes biológicos e ambientais no desenvolvimento neuropsicomotor em uma amostra de crianças de Canoas/RS. Ciência e Saúde Coletiva, 12(1), 181-190

Robins, D. (2008). Screening for autism spectrum disorders in primary care settings. The National Autistic Society,12(5), 481-500. 
Robins, D. L., Fein, D., Barton, M. L., \& Green, J. A. (2001). The Modified Checklist for Autism in Toddlers: an initial study investigating the early detection of autism and pervasive developmental disorders. Journal of Autism and Developmental Disorders, 31(2), 131-144.

Saulnier, C., Quiembach, L. E., \& Klin, A. (2011). Avaliação Clínica De Crianças Com Transtornos do Espectro do Autismo. In J. S. Schwartzman \& C. A. Araujo (Eds.), Transtornos do Espectro do Autismo (pp. 159-172). São Paulo: Memnon.

Saccani, R., Brizola, E., Giordani, A. P., Bach, S., Resende, T. L., \& Almeida, C. S. (2007). Avaliação do desenvolvimento neuropsicomotor em crianças de um bairro da periferia de Porto Alegre. Scientia Médica, 17(3), 130-137.

Teixeira, M. C. T. V., Mecca, T. P., Velloso, R. L., Bravo, R. B., Ribeiro, S. H. B., Mercadante, M. T., \& Paula, C. S. (2010), Literatura científica brasileira sobre transtornos do espectro autista. Revista da Associação Médica Brasileira, 56(5), 607-614.
Vieira, M. E., Ribeiro, F. V., \& Formiga, C. K. M. R. (2009). Principais instrumentos de avaliação de desenvolvimento da criança de zero a dois anos de idade. Revista Movimenta, $2(1), 23-31$.

Virués-Ortega, J. (2010). Applied behavior analytic intervention for autism in early childhood: Meta-analysis, meta-regression and dose-response meta-analysis of multiple outcomes. Clinical Psychology Review, 30(4), 387-399.

Wong, V., Hui, L. H., Lee, W. C., Leung, L. S., Ho, P. K., Lau, W. L., \& Chung, B. (2004). A modified screening tool for autism Checklist for Autism in Toddlers HAT-23 for Chinese children. Pediatrics, 114(2), 166-176.

Zeppone, S. C., Volpon, L. C., \& Del Ciampo, L. A. (2012). Monitoramento do desenvolvimento infantil realizado no Brasil. Revista Paulista de Pediatria, 30(4), 594-599.

Recebido em 22.11.2013

Primeira decisão editorial em 14.04.2014

Versão final em 18.08.2014

Aceito em 15.01.2015 